Kathryn E. Hare and Saroosh Yazdani, Department of Pure Mathematics, University of Waterloo, Waterloo, Ont., N2J 3G1, Canada. e-mail:

kehare@uwaterloo.ca

\title{
QUASI SELF-SIMILARITY AND MULTIFRACTAL ANALYSIS OF CANTOR MEASURES*
}

\begin{abstract}
We examine quasi self-similarity for Cantor measures on Cantor sets. A characterization is obtained in terms of the ratios of dissection of the Cantor set. The multifractal theory of Cantor measures is studied, extending the analysis for quasi self-similar measures.
\end{abstract}

\section{Introduction}

The main problem in multifractal theory is to estimate the size, typically the Hausdorff or packing dimension, of

$$
X(\alpha) \equiv\left\{x \in \operatorname{supp} \mu: \lim _{r \rightarrow 0} \frac{\log \mu(B(x, r))}{\log r}=\alpha\right\},
$$

where $\mu$ is a probability measure. The sets $X(\alpha)$ can be viewed as a decomposition of supp $\mu$ into a family of subfractals, the dimensions of these subfractals $X(\alpha)$ being known as the multifractal spectrum of $\mu$. The heuristic arguments developed by physicists and mathematicians (for an extensive bibliographic list see [4] and [6]) suggest that these dimensions should be equal to the Legendre transform of a suitable function. Olsen in [6] developed mathematical theories to put these arguments on a firm foundation and detailed analysis of the multifractal spectrum has been carried out for particular examples, such as

Key Words: Cantor sets, quasi self-similarity, dimension, multifractal spectrum

Mathematical Reviews subject classification: Primary 28A80; Secondary 28A78

Received by the editors April 10, 2000

*This research is supported in part by NSERC 
self-affine measures (see [5], [7]), self-similar measures ([2], [3]), and measures on cookie cutters $([9])$.

Recently, the multifractal spectrum was determined for quasi self-similar measures in metric spaces by O'Neil [8]. These are measures for which there exists a map from small parts of the space to itself and vice versa, which distorts distances and the measure in a bi-Lipschitz fashion.

In this paper the quasi self-similarity properties of Cantor sets and Cantor measures are investigated. We introduce a variation of quasi self-similarity for Cantor sets and characterize this property in terms of the ratios of dissection of the Cantor set. Sets which have this quasi self-similarity property are seen to have positive Hausdorff dimension. We also show that this variation coincides with the more general definition for most Cantor sets.

The fine multifractal structure of Cantor measures is then analyzed. We extend the work of [8] by showing that the multifractal spectrum of many Cantor measures is the Legendre transform of a function of the ratios of dissection of the Cantor set. The class of measures to which our work applies is larger than the class of quasi self-similar Cantor measures.

\section{Notation and Definitions}

\subsection{Cantor Sets}

By a Cantor set we mean a compact, totally disconnected, perfect subset of $[0,1]$ which can be constructed in a similar fashion to the classical middle third Cantor set. We remove from [0,1] a non-empty, centered, open interval, leaving two closed intervals of equal, positive length called the Cantor intervals of step one. The ratio between the length of the Cantor intervals of step one and $[0,1]$ is called the ratio of dissection of step one. A similar operation is performed on each Cantor interval of step one, producing the (four) closed equal-length Cantor intervals of step 2 and the ratio of dissection at step two. Repeating this construction yields a decreasing sequence of closed sets whose intersection is a Cantor set.

It is convenient to label the elements of the construction of the Cantor set by $W$, the set of binary words of finite length,

$$
W=\left\{w=w_{1} w_{2} \cdots w_{n}: w_{i} \in\{0,1\}, n \in \mathbb{N}\right\} \bigcup\{e\},
$$

where $e$ is the empty word. We let $I_{e}=[0,1]$. More generally, if the length of the word $w \in W$ (written $|w|)$ is $k$, then we denote by $I_{w 0}$ and $I_{w 1}$ the left and right Cantor intervals of step $k+1$ obtained after removing the open interval (or gap) $G_{w}$ of step $k$, from $I_{w}$, a Cantor interval of step $k$. We let $T$ denote 
the truncation operator, $I_{T(w i)}=I_{w}$, for $i=0,1$. The ratio of dissection at step $k$ will be denoted $r_{k}$ and is equal to $\left|I_{w i}\right| /\left|I_{w}\right|$ for any word $w$ of length $|w|=k-1$, and $i=0,1$. The Cantor set $C_{\left\{r_{k}\right\}}$ is the set $\bigcap_{k \in \mathbb{N}} \bigcup_{|w|=k} I_{w}$, and is uniquely determined by the ratios of dissection $\left\{r_{k}\right\}$. The classical middle third Cantor set is the case when the ratios of dissection are all equal to $1 / 3$.

We will also write $I_{k}$ and $G_{k}$ for Cantor intervals or gaps of step $k$ when only the step is relevant. Lastly, we note that sometimes it is convenient to write $I_{w}$ for the Cantor interval $I_{w}$ intersected with the Cantor set; the meaning should be clear from the context.

\subsection{Quasi Self-Similar Measures}

The measure which arises as the invariant measure from an iterated function system of similarity transforms is referred to as a self-similar measure; the attractor is a self-similar set. These sets and measures have been extensively studied (c.f. [4] and the references cited therein). Generalizing this idea, O'Neil in [8] defined the notion of a quasi self-similar measure on a compact metric space $X$.

Throughout the paper we let $B(x, d)$ denote the closed ball in $X$ with center $x \in X$ and radius $d$.

Definition 1. ([8]) Let $\mu$ be a Borel probability measure supported on $X$. We say that $\mu$ is $m$-quasi self-similar by balls from below on the space $X$ if for all $x \in X$ and balls $B(x, d)$ there exist mappings $\Phi: B(x, d) \rightarrow X$ (called the quasi self-similarity maps) such that whenever $B(y, s) \subset B(x, d)$ we have

$$
B\left(\Phi(y), m^{-1} \frac{s}{d}\right) \subset \Phi(B(y, s)) \subset B\left(\Phi(y), m \frac{s}{d}\right),
$$

and whenever $A \subset B(x, d)$ is a Borel set, then

$$
m^{-1} \frac{\mu(A)}{\mu(B(x, d))} \leq \mu(\Phi(A)) \leq m \frac{\mu(A)}{\mu(B(x, d))} .
$$

We say that $\mu$ is $m$-quasi self-similar by balls from above on $X$ if for all $x \in X$ and balls $B(x, d)$ there exist mappings $\Phi: X \rightarrow B(x, d)$ such that if $\rho \leq$ diameter $X$, then for all $z \in X$ one can find $\zeta \in B(x, d)$ such that

$$
B\left(\zeta, m^{-1} \rho d\right) \subset \Phi(B(z, \rho)) \subset B(\zeta, m \rho d),
$$

and if $A \subset X$ is Borel, then

$$
m^{-1} \mu(A) \leq \frac{\mu(\Phi(A))}{\mu(B(x, d))} \leq m \mu(A) .
$$


The measure $\mu$ is quasi self-similar by balls if it is both quasi self-similar from above and below on $X$.

For Cantor sets it is natural to to study a variant of quasi self-similarity where balls (contained in the Cantor set) are replaced in the definition of quasi self-similarity by Cantor intervals.

Definition 2. We say that a Borel probability measure $\mu$ on a Cantor set $C=C_{\left\{r_{k}\right\}}$ is $m$-quasi self-similar by Cantor intervals from below on $C$ if for all Cantor intervals $I_{w}$ there exist mappings $\Phi: I_{w} \rightarrow C$ such that for all $I_{v} \subset I_{w}$ there exists $x \in I_{v}$ such that

$$
B\left(\Phi(x), m^{-1} \frac{\left|I_{v}\right|}{\left|I_{w}\right|}\right) \subset \Phi\left(I_{v}\right) \subset B\left(\Phi(x), m \frac{\left|I_{v}\right|}{\left|I_{w}\right|}\right),
$$

and for all Borel sets $A \subset I_{w}$,

$$
m^{-1} \frac{\mu(A)}{\mu\left(I_{w}\right)} \leq \mu(\Phi(A)) \leq m \frac{\mu(A)}{\mu\left(I_{w}\right)} .
$$

We say that a measure $\mu$ on a Cantor set $C$ is $m$-quasi self-similar by Cantor intervals from above on $C$ if for all Cantor intervals $I_{w}$ there exist mappings $\Phi: C \rightarrow I_{w}$ such that for all $z \in C$ there exists $\zeta \in I_{w}$ such that if $z \in I_{v}$, then

$$
B\left(\zeta, m^{-1}\left|I_{v}\right|\left|I_{w}\right|\right) \subset \Phi\left(I_{v}\right) \subset B\left(\zeta, m\left|I_{v}\right|\left|I_{w}\right|\right),
$$

and for all Borel sets $A \subset C$,

$$
m^{-1} \mu(A) \leq \frac{\mu(\Phi(A))}{\mu\left(I_{w}\right)} \leq m \mu(A) .
$$

A measure on a Cantor set will be said to be quasi self-similar by Cantor intervals if it is both quasi self-similar by Cantor intervals from below and above.

There are a natural class of Borel probability measures on Cantor sets to study.

Definition 3. Let $C=C_{\left\{r_{k}\right\}}$ be a Cantor set and $0<p<1$. A Borel probability measure $\mu=\mu_{p}$, supported on $C$, is called a $p$-Cantor measure on $C$ if for all finite words $w$ we have $\mu\left(I_{w 0}\right)=p \mu\left(I_{w}\right)$ and $\mu\left(I_{w 1}\right)=(1-$ p) $\mu\left(I_{w}\right)$.

If the finite word $w$ has $j$ zeros and $k$ ones, then $\mu\left(I_{w}\right)=p^{j}(1-p)^{k}$.

One easy observation, which we will use extensively, is that any ball $B(x, d)$, with center $x \in C$ and $d<\min \left\{\left|G_{k}\right|: k \leq n\right\}$, cannot intersect two Cantor intervals of step $n+1$ as these are separated by more than $d$. Hence $B(x, d)$ must be a subset of the step $n+1$ Cantor interval containing $x$. 


\section{Quasi Self-Similarity by Cantor Intervals}

The main result of this section will be a characterization of quasi self-similarity by Cantor intervals in terms of the ratios of dissection of the Cantor set. Before stating this we prove a preliminary result which is of independent interest.

Proposition 1. If the p-Cantor measure $\mu$ on $C_{\left\{r_{k}\right\}}$ is quasi self-similar by Cantor intervals, then $\inf _{k} r_{k}>0$.

Proof. Assume $\mu$ is $m$ quasi self-similar by Cantor intervals and $\inf _{k} r_{k}=0$. Pick $n$ such that $m \max (p, 1-p)^{n}<\min (p, 1-p)$, and choose $\varepsilon>0$ so that the gaps of any step $j \leq n-1$ are greater than $m \varepsilon$ in length. Then for each $z \in C$ the ball $B(z, m \varepsilon)$ is contained in some Cantor interval $I_{v}$ (depending on $z$ ) of step $n$.

Choose $k$ such that $r_{k}<\varepsilon$ and let $I_{w}$ be any Cantor interval of step $k$. Since $\mu$ is quasi self-similar from below by Cantor intervals there exists a map $\Phi: I_{T(w)} \rightarrow C$ such that for some $z \in I_{w}$,

$$
\Phi\left(I_{w}\right) \subseteq B\left(\Phi(z), \frac{m\left|I_{w}\right|}{\left|I_{T(w)}\right|}\right) \subseteq B(\Phi(z), m \varepsilon) \subseteq I_{v},
$$

and

$$
m^{-1} \frac{\mu\left(I_{w}\right)}{\mu\left(I_{T(w)}\right)} \leq \mu\left(\Phi\left(I_{w}\right)\right) \leq \mu\left(I_{v}\right) .
$$

This is a contradiction since $\mu\left(I_{v}\right) \leq \max (p, 1-p)^{n}$ and $\mu\left(I_{w}\right) / \mu\left(I_{T(w)}\right) \geq$ $\min (p, 1-p)$.

Theorem 2. Let $C=C_{\left\{r_{k}\right\}}$ be a Cantor set. There is a p-Cantor measure on $C$ which is quasi self-similar by Cantor intervals if and only if there exists $n$ such that for all integers $i, j$,

$$
n^{-1} \leq \frac{r_{1} \cdots r_{i}}{r_{1+j} \cdots r_{i+j}} \leq n
$$

Moreover, in this case every p-Cantor measure on $C$ is quasi self-similar by Cantor intervals.

Proof. First, assume (3.1) fails to be satisfied for any $n$ but that $C$ supports a $p$-Cantor measure which is $m$-quasi self-similar by Cantor intervals. By the previous proposition we may assume inf $r_{k} \equiv a>0$. Choose $k, l \in \mathbb{N}$ such that $2^{k} \geq m$ and $2^{l-1}>a^{-4 k}$. The choice of $k$ ensures that $\left|I_{k}\right| \leq m^{-1}$, therefore if $x \in C \bigcap I_{k}$, then $I_{k} \subseteq B\left(x, m^{-1}\right)$. 
Case 1. The ratio is unbounded from above: Obtain $i, j$ such that

$$
\frac{r_{1} \cdots r_{i}}{r_{1+j} \cdots r_{i+j}}>2^{l} m
$$

and consider any word $w$ of length $j$. There is a quasi self-similarity mapping $\Phi: I_{w} \rightarrow C$ such that for all words $v$ of length $i$, there is some $x_{v} \in C$ with

$$
B\left(x_{v}, m^{-1} \frac{\left|I_{w v}\right|}{\left|I_{w}\right|}\right) \subseteq \Phi\left(I_{w v}\right) \subseteq B\left(x_{v}, m \frac{\left|I_{w v}\right|}{\left|I_{w}\right|}\right) .
$$

But $m \frac{\left|I_{w v}\right|}{\left|I_{w}\right|}=m\left|I_{v}\right| \frac{r_{1+j} \ldots r_{i+j}}{r_{1} \ldots r_{i}}<\frac{\left|I_{v}\right|}{2^{l}}$. Thus

$$
\bigcup_{|v|=i} \Phi\left(I_{w v}\right) \subseteq \bigcup_{|v|=i} B\left(x_{v}, \frac{\left|I_{v}\right|}{2^{l}}\right)
$$

For each $v \in W$ chose the word $s(v)$ of minimal length such that $B\left(x_{v}, \frac{\left|I_{v}\right|}{2^{l}}\right) \supseteq$ $I_{s(v)}$. As $s(v)$ is minimal in length, $B\left(x_{v}, \frac{\left|I_{v}\right|}{2^{l}}\right)$ will intersect at most four intervals of step $|s(v)|$. We will write $I_{s(v)}^{*}$ for the union of these (at most) four intervals. The fact that $B\left(x_{v}, \frac{\left|I_{v}\right|}{2^{l}}\right) \supseteq I_{s(v)}$ means that

$$
\frac{r_{1} \cdots r_{i}}{2^{l}}=\frac{\left|I_{v}\right|}{2^{l}} \geq \frac{\left|I_{s(v)}\right|}{2}=\frac{r_{1} \cdots r_{|s(v)|}}{2},
$$

and this implies $2^{-l+1} \geq r_{i+1} \ldots r_{|s(v)|} \geq a^{|s(v)|-i}$. As $2^{l-1}>a^{-4 k}$ this can only be true if $|s(v)|>4 k+i$.

Taking $v$ to be the empty word $e$ and $I_{u}$ the step $k$ interval containing $x_{e}$, it follows from the choice of $k$ and (3.2) that $I_{u} \subseteq B\left(x_{e}, m^{-1}\right) \subseteq \Phi\left(I_{w}\right)$. Since $I_{w}=\bigcup_{|v|=i} I_{w v}$ this gives the inclusions

$$
I_{u} \subseteq \bigcup_{|v|=i} \Phi\left(I_{w v}\right) \subseteq \bigcup_{|v|=i} B\left(x_{v}, \frac{\left|I_{v}\right|}{2^{l}}\right) \subseteq \bigcup_{|v|=i} I_{s(v)}^{*} .
$$

But the set on the right is a union of at most $2^{i+2}$ intervals of step $|s(v)|$, while $I_{u}$ is the union of $2^{|s(v)|-k}$ such intervals. Since $s(v)>4 k+i$ this is a contradiction.

Case 2. The ratio is unbounded from below: The argument is similar to the first case. Select $i, j$ such that $\frac{r_{1} \cdots r_{i}}{r_{1+j} \cdots r_{i+j}}<\frac{1}{m 2^{l}}$. 
Let $w$ be a word of length $j$ and let $\Phi: C \rightarrow I_{w}$ be an $m$-quasi self-similarity map. For all words $v$ of length $i$ we have

$$
\Phi\left(I_{v}\right) \subseteq B\left(x_{v}, m\left|I_{v}\right|\left|I_{w}\right|\right) \subseteq B\left(x_{v}, \frac{\left|I_{w v}\right|}{2^{l}}\right) .
$$

The fact that $2^{k} \geq m$ also ensures that for some $u \in W$ of length $k \geq 1$, the Cantor interval $I_{w u}$ is contained in $B\left(x_{e}, m^{-1}\left|I_{w}\right|\right)$. Thus the quasi self-similarity property of $\Phi$ also implies that $B\left(x_{e}, m^{-1}\left|I_{w}\right|\right) \subseteq \Phi\left(I_{e}\right) \subseteq$ $\bigcup_{|v|=i} \Phi\left(I_{v}\right)$, and therefore $I_{w u} \subseteq \bigcup_{|v|=i} B\left(x_{v}, \frac{\left|I_{w v}\right|}{2^{l}}\right)$.

Choose $s(v)$ of minimal length such that $B\left(x_{v}, \frac{\left|I_{w v}\right|}{2^{l}}\right) \supseteq I_{s(v)}$; then $\frac{\left|I_{w v}\right|}{2^{l-1}} \geq\left|I_{s(v)}\right|$ and $B\left(x_{v}, \frac{\left|I_{w v}\right|}{2^{l}}\right) \subseteq I_{s(v)}^{*}$ where $I_{s(v)}^{*}$ is a union of at most four step $s(v)$ intervals. Similar arguments to those used in the first case show that $|s(v)|>i+j+4 k$, which leads to the contradiction $I_{w u} \subseteq \bigcup_{|v|=i} I_{s(v)}^{*}$. This proves inequality (3.1) holds when $C$ supports a $p$-Cantor measure quasi self-similar by Cantor intervals.

Now we will show that if (3.1) holds, then every $p$-Cantor measure is quasi self-similar by Cantor intervals from below. The proof for quasi self-similar from above is similar. Given a Cantor interval $I_{w}$ we define a map $\Phi: I_{w} \rightarrow C$ by the rule $\Phi\left(I_{w v}\right)=I_{v}$ and extend this to elements of $I_{w}$ in the natural way. First, we will show that there exists $m$ such that for all $v \in W$ there is some $x \in I_{w v}$ with

$$
B\left(\Phi(x), m^{-1} \frac{\left|I_{w v}\right|}{\left|I_{w}\right|}\right) \subseteq \Phi\left(I_{w v}\right) \subseteq B\left(\Phi(x), m \frac{\left|I_{w v}\right|}{\left|I_{w}\right|}\right) .
$$

If one takes $x$ such that $\Phi(x)$ is an endpoint of $G_{v}$, then these inclusions will be satisfied if there exists some $m$ (independent of $v$ ) with $\left|I_{v}\right| \leq m \frac{\left|I_{w v}\right|}{\left|I_{w}\right|}$ and $\left|I_{v 0}\right| \geq m^{-1} \frac{\left|I_{w v}\right|}{\left|I_{w}\right|}$. Upon simplifying, these inequalities reduce to

$$
\left(r_{|v|+1} m\right)^{-1} \leq \frac{r_{1} \cdots r_{|v|}}{r_{1+|w|} \cdots r_{|v|+|w|}} \leq m
$$

Thus if there exists $m \geq n$ such that $\left(r_{|v|+1} m\right)^{-1} \leq n^{-1}$ for all $v \in W$, then we are done. However, if one takes $i=1$, and $j=|v|$ in (3.1), the inequality $n / r_{|v|+1} \leq n^{2} / r_{1}$ is derived. Thus if $m \geq n^{2} / r_{1}$, we have the desired inclusions. 
If $\mu$ is any $p$-Cantor measure, then since $\mu\left(I_{w v}\right) / \mu\left(I_{w}\right)=\mu\left(I_{v}\right)$, the inequalities $\frac{m^{-1} \mu(A)}{\mu\left(I_{w}\right)} \leq \mu(\Phi(A)) \leq \frac{m \mu(A)}{\mu\left(I_{w}\right)}$ hold for all Cantor intervals $A \subseteq I_{w}$ and any $m \geq 1$. As these sets generate the Borel sets in $C$ the same relationship holds for all Borel sets $A$. This completes the proof that $\mu$ is quasi self-similar from below.

Corollary 3. If $C$ supports a p-Cantor measure which is quasi self-similar by Cantor intervals, then every p-Cantor measure is quasi self-similar by Cantor intervals.

The Hausdorff dimension of the Cantor set $C_{\left\{r_{k}\right\}}$ is known ([1]) to equal

$$
\liminf _{k \rightarrow \infty} \frac{\log 2}{\frac{1}{k}\left|\log r_{1} \cdots r_{k}\right|},
$$

hence it is of interest to know how condition (3.1) compares to convergence properties of the geometric means of the ratios of dissection. We have the following relationships.

Proposition 4. (i) If condition (3.1) is satisfied, i.e. there exists $n$ such that for all integers $i, j n^{-1} \leq \frac{r_{1} \cdots r_{i}}{r_{1+j} \cdots r_{i+j}} \leq n$, then $\left(r_{1} \cdots r_{k}\right)^{1 / k}$ converges (to a non-zero limit). The converse is not true.

(ii) Condition (3.1) will be satisfied for some $n$ if there exist $0<a, b, \lambda<\infty$ such that $a \leq \frac{r_{1} \ldots r_{k}}{\lambda^{k}} \leq b$ for all $k$.

Proof. (i) Let $H_{k}=r_{1} \cdots r_{k}$ and $S_{k}=\log \left(H_{k}\right)$. Condition (3.1) implies that for all $i, j$ we have $n^{-1} \leq \frac{H_{i} H_{j}}{H_{i+j}} \leq n$. Thus if we let $\delta=\log n$ and rearrange terms we obtain

$$
-\delta+S_{i}+S_{j} \leq S_{i+j} \leq \delta+S_{i}+S_{j}
$$

It suffices to prove that $\left\{S_{j} / j\right\}$ forms a Cauchy sequence. First note that an induction argument (on $i$ ) using (3.3) shows that $-i \delta+i S_{j} \leq S_{i j} \leq i \delta+i S_{j}$. Manipulating this inequality gives $-(i+j) \delta+i S_{j} \leq j S_{i} \leq(i+j) \delta+i S_{j}$, and thus $\left|\frac{S_{i}}{i}-\frac{S_{j}}{j}\right| \leq\left(\frac{1}{i}+\frac{1}{j}\right) \delta$. Clearly this implies that $\left\{S_{j} / j\right\}$ is a Cauchy
sequence.

To see that the converse is not true note that $\left(r_{1} \cdots r_{k}\right)^{1 / k}$ can converge, and yet inf $r_{k}=0$. From Proposition 1 we know that condition (3.1) cannot be satisfied in this case.

(ii) One can easily check that (3.1) is satisfied with $n=\max \left(b^{2} / a, b / a^{2}\right)$. 
Corollary 5. (i) If $C$ supports a p-Cantor measure which is quasi self-similar by Cantor intervals, then $C$ has positive Hausdorff dimension.

(ii) There are Cantor sets of positive Hausdorff dimension which do not support any quasi self-similar p-Cantor measures.

Corollary 6. Every p-Cantor measure on $C_{\left\{r_{k}\right\}}$ is quasi self-similar by Cantor intervals if there exist $0<a, b, \lambda<\infty$ such that $a \leq \frac{r_{1} \ldots r_{k}}{\lambda^{k}} \leq b$ for all $k$.

\section{Equivalence of Quasi Self-Similarity by Balls and Can- tor Intervals}

In this section we will show that for $p$-Cantor measures supported on Cantor sets with ratios of dissection bounded away from $1 / 2$, quasi self-similarity by balls or Cantor intervals coincide, but that they need not coincide in general. The assumption on the ratios of dissection is important because it ensures that balls and appropriate Cantor intervals are comparable. (It effectively replaces the strong separation condition.) One formulation of this is given in the lemma below.

Lemma 7. Suppose $C=C_{\left\{r_{k}\right\}}$ is a Cantor set with $\sup r_{k}<1 / 2$. There exists an integer $N$ such that if $x \in I_{w}$, then $I_{w} \subseteq B\left(x,\left|I_{w}\right|\right) \subseteq I_{T^{N}(w)}$. Similarly, if $x \in C$ and $d>0$, then there is some $w \in W$ such that $x \in I_{w} \subseteq$ $B(x, d) \subseteq I_{T^{N}(w)}$.

Proof. The assumption that $\sup r_{k}<1 / 2$ is equivalent to the statement that there exists $\delta>0$ (namely, $\delta=\sup \left(1-2 r_{k}\right)$ ) such that $\left|G_{v}\right| \geq \delta\left|I_{v}\right|$ for all $v \in W$. Choose $N$ such that $2^{-N+1}<\delta$. As $\left|I_{T^{N}(w)}\right| \geq 2^{N}\left|I_{w}\right|$, this ensures that $\left|I_{w}\right|<\left|G_{v}\right|$ for any $v$ such that $|v| \leq|w|-N$. Thus $B\left(x,\left|I_{w}\right|\right)$ must be contained in $I_{T^{N}(w)}$.

To prove the second statement, choose $k$ such that $r_{1} \cdots r_{k} \leq d<r_{1} \cdots r_{k-1}$, let $I_{w}$ be the Cantor interval of step $k$ containing $x$, and argue as in the first paragraph.

Theorem 8. Suppose $C=C_{\left\{r_{k}\right\}}$ is a Cantor set with $\sup r_{k}<1 / 2$. Any p-Cantor measure on $C$ is quasi self-similar by balls if and only if it is quasi self-similar by Cantor intervals.

Proof. Throughout the proof we fix $\delta$ with $0<\delta<\sup \left(1-2 r_{k}\right)$. First, assume $\mu$ is $m$-quasi self-similar by balls on $C$. We will begin by establishing that $\mu$ is quasi self-similar by Cantor intervals from below, so let $I_{w}$ be a Cantor interval and denote by $x$ an endpoint of $I_{w}$. Let $\Phi: B\left(x,\left|I_{w}\right|\right) \rightarrow C$ 
be a quasi self-similarity map from below, suppose $I_{v} \subseteq I_{w}$ and assume $z$ is the endpoint of $I_{v}$ closest to $x$. Because $\delta\left|I_{v}\right|$ is less than the size of any gap of step $j \leq|v|, B\left(z, \delta\left|I_{v}\right|\right) \subseteq I_{v}$. The choice of $z$ ensures that

$$
I_{v} \subseteq B\left(z,\left|I_{v}\right|\right) \subseteq B\left(x,\left|I_{w}\right|\right) ;
$$

so the definition of an $m$-quasi self-similar map yields the inclusions

$$
\begin{aligned}
B\left(\Phi(z), \frac{m^{-1} \delta\left|I_{v}\right|}{\left|I_{w}\right|}\right) & \subseteq \Phi\left(B\left(z, \delta\left|I_{v}\right|\right)\right) \subseteq \Phi\left(I_{v}\right) \\
& \subseteq \Phi\left(B\left(z,\left|I_{v}\right|\right)\right) \subseteq B\left(\Phi(z), \frac{m\left|I_{v}\right|}{\left|I_{w}\right|}\right) .
\end{aligned}
$$

Moreover, for all Borel sets $A \subseteq B\left(x,\left|I_{w}\right|\right)$ we have

$$
\frac{m^{-1} \mu(A)}{\mu\left(B\left(x,\left|I_{w}\right|\right)\right)} \leq \mu(\Phi(A)) \leq \frac{m \mu(A)}{\mu\left(B\left(x,\left|I_{w}\right|\right)\right)} .
$$

By the lemma there is a constant $N$ (independent of $x$ and $w$ ) such that $I_{w} \subseteq B\left(x,\left|I_{w}\right|\right) \subseteq I_{T^{N}(w)}$. Thus $\mu\left(B\left(x,\left|I_{w}\right|\right)\right)$ is comparable to $\mu\left(I_{w}\right)$. These observations show that for a suitable constant $m_{1}$ and all Borel sets $A \subseteq I_{w}$, we have

$$
B\left(\Phi(z), \frac{m_{1}^{-1}\left|I_{v}\right|}{\left|I_{w}\right|}\right) \subseteq \Phi\left(I_{v}\right) \subseteq B\left(\Phi(z), \frac{m_{1}\left|I_{v}\right|}{\left|I_{w}\right|}\right),
$$

and

$$
\frac{m_{1}^{-1} \mu(A)}{\mu\left(I_{w}\right)} \leq \mu(\Phi(A)) \leq \frac{m_{1} \mu(A)}{\mu\left(I_{w}\right)} .
$$

Thus $\Phi$ restricted to $I_{w}$ is a suitable map and therefore $\mu$ is quasi self-similar by Cantor intervals from below.

To see that $\mu$ is quasi self-similar by Cantor intervals from above we again start with a Cantor interval $I_{w}$. Choose $x$ in the Cantor set belonging to $I_{w}$ and let $\Phi: C \rightarrow B\left(x, \delta\left|I_{w}\right|\right) \subseteq I_{w}$ be a $m$-quasi self-similarity map from above. If $z \in C \cap I_{v}$, then choosing $\zeta$ as in the definition of quasi self-similarity by balls we obtain the inclusions

$$
\begin{aligned}
B\left(\zeta, m^{-1} \delta\left|I_{v}\right| \delta\left|I_{w}\right|\right) & \subseteq \Phi\left(B\left(z, \delta\left|I_{v}\right|\right)\right) \subseteq \Phi\left(I_{v}\right) \\
& \subseteq \Phi\left(B\left(z,\left|I_{v}\right|\right)\right) \subseteq B\left(\zeta, m\left|I_{v}\right| \delta\left|I_{w}\right|\right) .
\end{aligned}
$$

If $N$ is chosen such that $2^{-N} \leq \delta$, then $B\left(x, \delta\left|I_{w}\right|\right)$ will contain the step $|w|+N$ Cantor subinterval of $I_{|w|}$ which contains $x$. This ensures that $\mu\left(B\left(x, \delta\left|I_{w}\right|\right)\right)$ is comparable to $\mu\left(I_{w}\right)$, and similar arguments to those used in the earlier part of the proof imply that $\mu$ is quasi self-similar by Cantor intervals from above. 
Now assume $\mu$ is quasi self-similar by Cantor intervals on $C$. We will show quasi self-similarity by balls from below; the proof for quasi self-similarity from above is similar. Consider a ball $B(x, d)$ with center $x \in C$ and, as in the lemma, choose the Cantor interval $I_{w}$ and integer $N$ independent of $x$ and $d$ such that $\left|I_{w}\right| \leq d<\left|I_{T(w)}\right|$ and $x \in I_{w} \subseteq B(x, d) \subseteq I_{T^{N}(w)}$. Let $\Phi: I_{T^{2 N}(w)} \rightarrow C$ be a quasi self-similarity map from below and suppose $B(y, s) \subseteq B(x, d)$. Choose $v$ such that $I_{v} \subseteq B(y, s) \subseteq I_{T^{N}(v)}$. Since $I_{v} \subseteq$ $I_{T^{N}(w)}$, we have $I_{T^{N}(v)} \subseteq I_{T^{2 N}(w)}$, thus the quasi self-similarity properties of $\Phi$ imply that

$$
\begin{aligned}
B\left(\Phi(x), \frac{m^{-1}\left|I_{v}\right|}{\left|I_{T^{2 N}(w)}\right|}\right) & \subseteq \Phi\left(I_{v}\right) \subseteq \Phi(B(y, s)) \\
& \subseteq \Phi\left(I_{T^{N}(v)} \subseteq B\left(\Phi(x), \frac{m\left|I_{T^{N}(v)}\right|}{\left|I_{T^{2 N}(w)}\right|}\right) .\right.
\end{aligned}
$$

The assumption of quasi self-similarity by Cantor intervals ensures that the ratios of dissection of $C$ are bounded away from zero, hence $\left|I_{T^{2 N}(w)}\right|$ is comparable to $d$, and the lengths of $I_{v}$ and $I_{T^{N}(v)}$ are comparable to $s$. Also, $\mu\left(I_{T^{2 N}(w)}\right)$ and $\mu(B(x, d))$ are comparable, hence there is a constant $m_{1}$ such that

$$
B\left(\Phi(x), \frac{m_{1}^{-1} s}{d}\right) \subseteq \Phi(B(y, s)) \subseteq B\left(\Phi(x), \frac{m_{1} s}{d}\right),
$$

and for all Borel sets $A \subseteq B(x, d)$,

$$
\frac{m_{1}^{-1} \mu(A)}{\mu(B(x, d))} \leq \frac{m^{-1} \mu(A)}{\mu\left(I_{T^{2 N}(w)}\right)} \leq \mu(\Phi(A)) \leq \frac{m \mu(A)}{\mu\left(I_{T^{2 N}(w)}\right)} \leq \frac{m_{1} \mu(A)}{\mu(B(x, d))} .
$$

Thus $\Phi$ restricted to the ball $B(x, d)$ is a suitable quasi self-similarity function.

Corollary 9. Suppose $\sup _{k} r_{k}<1 / 2$. Then a p-Cantor measure on $C_{\left\{r_{k}\right\}}$ is quasi self-similar by balls if and only if there exists $n$ such that (3.1) holds for all $i, j$. If $C_{\left\{r_{k}\right\}}$ supports a p-Cantor measure which is quasi self-similar by Cantor intervals (or, equivalently, balls), then every $p$-Cantor measure is quasi self-similar by Cantor intervals and balls.

However, the two quasi self-similarity properties are not the same. To show this we will prove one additional property of quasi self-similarity by balls.

Proposition 10. Suppose $C$ is a Cantor set whose gaps decrease in length as the step increases. If there is a p-Cantor measure on $C$ which is quasi self-similar by balls, then $\sup _{k} r_{k}<1 / 2$. 
Proof. Suppose $\mu$ is $m$-quasi self-similar and choose $n$ such that

$$
m \max (p, 1-p)^{n}<\min (p, 1-p) .
$$

Assume to the contrary that $\sup _{k} r_{k}=1 / 2$. Then we can choose $k>n$ such that $\frac{m\left|G_{k-1}\right|}{\left|I_{k}\right|+\left|G_{k-1}\right|}=\frac{m\left(1-2 r_{k}\right)}{1-r_{k}}<\left|G_{n}\right|$. Set $s=\left|G_{k-1}\right|, d=\left|I_{k}\right|$ and let $x \in I_{k}$ be an endpoint of $G_{k-1}$. Clearly, $I_{k} \subseteq B(x, d) \subseteq I_{k-1}$. Since $\mu$ is quasi self-similar by balls, there exists a quasi self-similarity map $\Phi: B(x, d+s) \rightarrow C$ which has the property that for any $z \in B(x, d)$,

$$
\Phi(B(z, s)) \subseteq B\left(\Phi(z), \frac{m s}{r+s}\right) .
$$

The assumption of decreasing gap lengths ensures that any gap of the Cantor set, contained in $B(x, d)$, has length at most $s$. This means we can choose elements of the Cantor set, $z_{1}, \ldots, z_{m}$ in $B(x, d)$, such that the balls $B\left(z_{i}, s\right)$ cover $B(x, d)$ and for each $i, B\left(z_{i}, s\right) \bigcap B\left(z_{i+1}, s\right)$ is non-empty. But because $m s /(d+s)$ is less than the length of any gap of step at most $n$, $B\left(\Phi(z), \frac{m s}{d+s}\right)$ must be contained in a Cantor interval of step $n+1$. Consequently, $\Phi\left(B\left(z_{i}, s\right)\right) \subseteq I_{v_{i}}$ for some $v_{i} \in W$ of length $n+1$. Since $\Phi\left(B\left(z_{i}, s\right)\right)$ and $\Phi\left(B\left(z_{i+1}, s\right)\right)$ intersect non-trivially, the intervals $I_{v_{i}}$ intersect, and therefore coincide. Thus $\Phi(B(x, d)) \subseteq I_{v}$ for some $|v|=n+1$. The quasi selfsimilarity property also implies that

$$
\frac{m^{-1} \mu(B(x, d))}{\mu(B(x, d+s))} \leq \mu\left(\Phi(B(x, d)) \leq \mu\left(I_{v}\right) .\right.
$$

Finally, we note that $B(x, d+s) \subseteq I_{k-1}$, thus

$$
\frac{m^{-1} \mu(B(x, d))}{\mu(B(x, d+s))} \geq \frac{m^{-1} \mu\left(I_{k}\right)}{\mu\left(I_{k-1}\right)} \geq m^{-1} \min (p, 1-p) .
$$

But $n$ was chosen so that $\mu\left(I_{v}\right)<m^{-1} \min (p, 1-p)$ which is a contradiction.

An immediate corollary of this Proposition and Theorem 8 is the following.

Corollary 11. If $C$ is a Cantor set with decreasing gaps, then any p-Cantor measure which is quasi self-similar by balls is quasi self-similar by Cantor intervals. 
Corollary 12. A Cantor set with ratios of dissection increasing to $1 / 2$ does not support any p-Cantor measure quasi self-similar by balls.

Proof. Since the ratios are increasing, the gaps must be decreasing in size, and hence the proposition directly applies.

We can now give an example of a $p$-Cantor measure which is quasi selfsimilar by Cantor intervals but not balls. We do not know if there are $p$-Cantor measures which are quasi self-similar by balls but not by intervals.

Corollary 13. If $r_{k}=2^{-1-1 / k^{2}}$, then every $p$-Cantor measure on the Cantor set $C_{\left\{r_{k}\right\}}$ is quasi self-similar by Cantor intervals but not by balls.

Proof. One can easily check that $\left\{r_{k}\right\}$ satisfies (3.1). However, the ratios increase to $1 / 2$ and thus the no $p$-Cantor measure is quasi self-similar by balls.

\section{Multifractal Analysis of $p$-Cantor Measures}

In [8] O'Neil showed that the multifractal formalism developed by Olsen [6] could be applied to quasi self-similar measures. In this section we obtain similar results for a broad class of $p$-Cantor measures, including those which are not necessarily quasi self-similar, but are supported on Cantor sets where the geometric means of the ratios of dissection converge.

Throughout the section we will assume that $\sup r_{k}<1 / 2$, and $\mu$ will denote a $p$-Cantor measure where, without loss of generality, we will assume $p=\max (p, 1-p)$.

We begin our study of the multifractal structure of Cantor sets by recalling the definition of the pointwise local upper and lower dimensions of $\mu$ at $x \in C$ :

$$
\begin{aligned}
& \varlimsup_{\operatorname{dim}}^{l o c} \mu(x)=\limsup _{d \rightarrow 0} \frac{\log \mu(B(x, d))}{\log d} ; \\
& \underline{\operatorname{dim}}_{l o c} \mu(x)=\liminf _{d \rightarrow 0} \frac{\log \mu(B(x, d))}{\log d} .
\end{aligned}
$$

The pointwise local dimension of $\mu$ is $\operatorname{dim}_{l o c} \mu(x)=\lim _{d \rightarrow 0} \frac{\log \mu(B(x, d))}{\log d}$ if this limit exists.

To analyze these local dimensions we will make significant use of the fact that balls centered in $C$ and appropriate Cantor intervals have comparable measure. For instance, if $k$ is chosen such that $r_{1} \cdots r_{k} \leq d<r_{1} \cdots r_{k-1}$ and $I_{w}$ is the step $k$ Cantor interval containing $x \in C$, then we know from 
Lemma 7 that $I_{w} \subseteq B(x, d) \subseteq I_{T^{N}(w)}$, and hence $p_{w_{1}} \cdots p_{w_{k}} \leq \mu(B(x, d)) \leq$ $p_{w_{1}} \cdots p_{w_{k-N}}$. Thus

$$
\frac{\log p_{w_{1}} \cdots p_{w_{k-N}}}{\log r_{1} \cdots r_{k}} \leq \frac{\log \mu(B(x, d))}{\log d} \leq \frac{\log p_{w_{1}} \cdots p_{w_{k}}}{\log r_{1} \cdots r_{k-1}}
$$

Since $N$ can be chosen independently of $x$ and $d$, the limiting behavior (as $k \rightarrow \infty)$ of $\frac{\log p_{w_{1}} \ldots p_{w_{k-N}}}{\log r_{1} \ldots r_{k}}$ and $\frac{\log p_{w_{1}} \cdots p_{w_{k}}}{\log r_{1} \cdots r_{k-1}}$ are the same, and coincide with the limits of $\frac{\log \mu\left(I_{w}\right)}{\log \left|I_{w}\right|}=\frac{\log p_{w_{1}} \ldots p_{w_{k}}}{\log r_{1} \ldots r_{k}}$. This means that in the definition of pointwise local dimension we may replace balls by Cantor intervals, in other words, $\operatorname{dim}_{l o c} \mu(x)=\lim _{x \in I_{w} ;\left|I_{w}\right| \rightarrow 0} \frac{\log \mu\left(I_{w}\right)}{\log \left|I_{w}\right|}$.

Motivated by the fact that box dimensions are often easier to calculate than Hausdorff dimension, Olsen ([6] Sec 2.7) introduced the multifractal box dimensions. To define these we need the following terminology. A countable family of closed balls $\left(B\left(x_{i}, d\right)\right)_{i}$ is called a centered packing of $C$ if $x_{i} \in C$ and the balls are pairwise disjoint. For $q \in \mathbb{R}$, and $d>0$ set

$$
S_{d}^{q}=\sup \left\{\sum_{i} \mu\left(B\left(x_{i}, d\right)\right)^{q}:\left(B\left(x_{i}, d\right)\right)_{i} \text { is a centred packing of } C\right\} .
$$

The upper and lower multifractal $q$-box dimensions, $C^{q}$ and $C_{q}$, are given by

$$
C^{q}=\limsup _{d \rightarrow 0} \frac{\log S_{d}^{q}}{|\log d|} \text { and } C_{q}=\liminf _{d \rightarrow 0} \frac{\log S_{d}^{q}}{|\log d|} .
$$

The comparison between measures of balls and Cantor intervals enables us to easily calculate these as we demonstrate below.

Proposition 14. There are constants $a=a(q), b=b(q)$ such that if $r_{1} \cdots r_{k} \leq$ $d<r_{1} \cdots r_{k-1}$, then $a\left(p^{q}+(1-p)^{q}\right)^{k} \leq S_{d}^{q} \leq b\left(p^{q}+(1-p)^{q}\right)^{k}$.

Proof. Let $\left(B\left(x_{i}, d\right)\right)_{i}$ be a centered packing of $C$. Let $I_{w}\left(x_{i}\right)$ be the Cantor interval of step $k$ containing $x_{i}$, so $I_{w}\left(x_{i}\right) \subseteq B\left(x_{i}, d\right) \subseteq I_{T^{N}(w)}\left(x_{i}\right)$. The balls $B\left(x_{i}, d\right)$ are disjoint by definition, thus the intervals $I_{w}\left(x_{i}\right)$ are distinct. Since each step $k-N$ interval contains $2^{N}$ step $k$ subintervals, a step $k-N$ interval can be the interval $I_{T^{N}(w)}\left(x_{i}\right)$ for at most $2^{N}$ choices of $x_{i}$. Hence

$$
\begin{aligned}
\sum_{i} \mu\left(B\left(x_{i}, d\right)\right)^{q} & \leq 2^{N} \sum_{|w|=k-N}\left(p_{w_{1}} \ldots p_{w_{k-N}}\right)^{q} \\
& =2^{N}\left(p^{q}+(1-p)^{q}\right)^{k-N} \equiv b\left(p^{q}+(1-p)^{q}\right)^{k} .
\end{aligned}
$$


To obtain the reverse inequality choose $N$ such that $\left|G_{T^{j}(w)}\right| \geq\left|I_{T(w)}\right|$ for all $j \geq N$ and for all $w \in W$. Let $x_{1}, \ldots, x_{2^{k-N}}$ denote the left hand endpoints of the step $k-N$ Cantor intervals. Since these step $k-N$ intervals are separated by gaps of at least $d$, the balls $B\left(x_{i}, d\right), i=1, \ldots, 2^{k-N}$, must be disjoint and hence form a centered packing of $C$. Because $d \geq r_{1} \cdots r_{k}$, $B\left(x_{i}, d\right)$ contains the step $k$ interval having $x_{i}$ as its left endpoint. Thus

$$
\begin{aligned}
\sum \mu\left(B\left(x_{i}, d\right)\right)^{q} & \geq \sum_{|w|=k-N} p^{N q}\left(p_{w_{1}} \cdots p_{w_{k-N}}\right)^{q} \\
& =p^{N q}\left(p^{q}+(1-p)^{q}\right)^{k-N}=a\left(p^{q}+(1-p)^{q}\right)^{k} .
\end{aligned}
$$

Corollary 15. The upper and lower multifractal q-box dimensions have the values

$$
C_{q}=\liminf _{k \rightarrow \infty} \frac{\log \left(p^{q}+(1-p)^{q}\right)}{\left|\frac{1}{k} \log r_{1} \cdots r_{k}\right|} ; C^{q}=\limsup _{k \rightarrow \infty} \frac{\log \left(p^{q}+(1-p)^{q}\right)}{\left|\frac{1}{k} \log r_{1} \cdots r_{k}\right|}
$$

Proof. This is a routine consequence of the proposition and the definition of the multifractal dimensions.

For $\alpha \in \mathbb{R}$ set

$$
\begin{aligned}
X^{\alpha} & =\left\{x \in C: \overline{\operatorname{dim}}_{l o c} \mu(x)=\alpha\right\}, \\
X_{\alpha} & =\left\{x \in C: \underline{\operatorname{dim}}_{l o c} \mu(x)=\alpha\right\}, \\
X(\alpha) & =\left\{x \in C: \operatorname{dim}_{l o c} \mu(x)=\alpha\right\}=X_{\alpha} \bigcap X^{\alpha} .
\end{aligned}
$$

These sets can be viewed as giving a multifractal decomposition of the Cantor set $C$ relative to the measure $\mu$, and the aim of the fine approach to multifractal analysis is to determine their dimensions. We will follow the method of [4], Sec. 11.2 by showing the existence of probability measures concentrated on these sets.

For this, define $\beta_{i}=\beta_{i}(q)$ by $\left(p^{q}+(1-p)^{q}\right) r_{i}^{\beta_{i}}=1$ and define a set function $v=v_{q}$ on the Cantor intervals by the rule $v\left(I_{w}\right)=\left(p_{w_{1}} \cdots p_{w_{k}}\right)^{q} r_{1}^{\beta_{1}} \cdots r_{k}^{\beta_{k}}$ if $|w|=k$. Notice that $v\left(I_{w 0}\right)+v\left(I_{w 1}\right)=v\left(I_{w}\right)$ and consequently $v$ extends to a probability measure supported on $C$. Since $\log \prod_{i=1}^{k} r_{i}^{\beta_{i}}=-k \log \left(p^{q}+(1-p)^{q}\right)$, the reader can readily use the definition of $\beta_{i}$ to verify that when $|w|=k$,

$$
\frac{\log v\left(I_{w}\right)}{\log \left|I_{w}\right|}=q \frac{\log \mu\left(I_{w}\right)}{\log \left|I_{w}\right|}+\frac{\log \left(p^{q}+(1-p)^{q}\right)}{\left|\frac{1}{k} \log r_{1} \ldots r_{k}\right|} .
$$


As with $\mu$, the local behavior of $v$ is the same whether one considers balls or Cantor intervals, thus we obtain

$$
\begin{aligned}
\underline{\operatorname{dim}}_{l o c} v(x) & =\liminf _{x \in I_{w} ;\left|I_{w}\right| \rightarrow 0} \frac{\log v\left(I_{w}\right)}{\log \left|I_{w}\right|} \\
& =\liminf \left(q \frac{\log \mu\left(I_{w}\right)}{\log \left|I_{w}\right|}+\frac{\log \left(p^{q}+(1-p)^{q}\right)}{\left|\frac{1}{k} \log r_{1} \ldots r_{k}\right|}\right) \\
& \geq \liminf q \frac{\log \mu\left(I_{w}\right)}{\log \left|I_{w}\right|}+C_{q} .
\end{aligned}
$$

Notice the inequality is an equality if $\lim _{x \in I_{w} ;\left|I_{w}\right| \rightarrow 0} \frac{\log \mu\left(I_{w}\right)}{\log \left|I_{w}\right|}$ exists, and in this case we have $\underline{\operatorname{dim}}_{l o c} v(x)=q \lim _{x \in I_{w} ;\left|I_{w}\right| \rightarrow 0} \frac{\log \mu\left(I_{w}\right)}{\log \left|I_{w}\right|}+C_{q}$. Similarly, $\overline{\operatorname{dim}}_{l o c} v(x) \leq \lim \sup q \frac{\log \mu\left(I_{w}\right)}{\log \left|I_{w}\right|}+C^{q}$, again with equality if $\lim \frac{\log \mu\left(I_{w}\right)}{\log \left|I_{w}\right|}$ exists. Set

$$
\alpha^{*} \equiv \alpha^{*}(q)=\frac{-\left(p^{q} \log p+(1-p)^{q} \log (1-p)\right)}{\left(p^{q}+(1-p)^{q}\right) \lim \sup _{k}\left|\frac{1}{k} \log r_{1} \cdots r_{k}\right|}
$$

and

$$
\alpha_{*} \equiv \alpha_{*}(q)=\frac{-\left(p^{q} \log p+(1-p)^{q} \log (1-p)\right)}{\left(p^{q}+(1-p)^{q}\right) \lim \inf _{k}\left|\frac{1}{k} \log r_{1} \cdots r_{k}\right|} .
$$

We are now ready to state the technical result from which we can determine bounds on the Hausdorff and packing dimensions of $X_{\alpha^{*}} \bigcap X^{\alpha_{*}}$.

Theorem 16. There exists a probability measure $v$ concentrated on $X_{\alpha^{*}} \bigcap X^{\alpha_{*}}$ which has the property that for $v$ a.e. $x$ in $X_{\alpha^{*}} \cap X^{\alpha_{*}}$ :

(i) If $q \geq 0$, then $\underline{\operatorname{dim}}_{l o c} v(x) \geq q \alpha^{*}+C_{q}$ and $\overline{\operatorname{dim}}_{l o c} v(x) \leq q \alpha_{*}+C^{q}$;

(ii) If $q \leq 0$, then $\underline{\operatorname{dim}}_{l o c} v(x) \geq q \alpha_{*}+C_{q}$ and $\overline{\operatorname{dim}}_{l o c} v(x) \leq q \alpha^{*}+C^{q}$.

The proof of the theorem can be obtained in a similar fashion to [4], Prop. 11.4 once we have established the following lemma.

Lemma 17. Let $\varepsilon>0, s \in \mathbb{R}$ and let $\delta>0$ be small. Let

$$
\alpha_{s} \equiv \alpha_{s}(q)=\frac{-\left(p^{q} \log p+(1-p)^{q} \log (1-p)\right)}{\left(p^{q}+(1-p)^{q}\right) s},
$$


and for $k \in \mathbb{N}$ set $\Phi_{ \pm}\left(\alpha_{s}\right)=\log \prod_{i=1}^{k}\left(p^{q \pm \delta}+(1-p)^{q \pm \delta}\right) r_{i}^{\beta_{i}+\left(\varepsilon \mp \alpha_{s}\right) \delta}$. Then

$$
\Phi_{ \pm}\left(\alpha_{s}\right) \leq k\left(\mp \alpha_{s} s \delta+O\left(\delta^{2}\right)-\varepsilon \delta \log 2 \pm \frac{\alpha_{s}}{k} \delta \sum_{i=1}^{k}\left|\log r_{i}\right|\right)
$$

If $t=\limsup \frac{1}{k} \sum_{i=1}^{k}\left|\log r_{i}\right|$, then there is a constant c such that $\Phi_{+}\left(\alpha_{t}\right) \leq$ $-k \delta c \varepsilon$ for all but finitely many $k$, and $\Phi_{-}\left(\alpha_{t}\right) \leq-k \delta c \varepsilon$ for infinitely many $k$.

If $t=\liminf \frac{1}{k} \sum_{i=1}^{k}\left|\log r_{i}\right|$, then there is a constant c such that $\Phi_{+}\left(\alpha_{t}\right) \leq$ $-k \delta c \varepsilon$ for infinitely many $k$, and $\Phi_{-}\left(\alpha_{t}\right) \leq-k \delta c \varepsilon$ for all but finitely many $k$.

Proof. This is essentially a calculus exercise. Using the first order Taylor expansion of $\left(p^{x}+(1-p)^{x}\right)$ at $x=q$ and the fact that $-\log \left(p^{q}+(1-p)^{q}\right)=$ $\beta_{i} \log r_{i}$, we readily obtain

$$
\begin{aligned}
\Phi_{ \pm}\left(\alpha_{s}\right)= & \sum_{1}^{k}\left(\log \left(p^{q \pm \delta}+(1-p)^{q \pm \delta}\right)-\log \left(p^{q}+(1-p)^{q}\right)\right) \\
& +\delta\left(\varepsilon \mp \alpha_{s}\right) \sum_{i=1}^{k} \log r_{i} \\
= & \pm \delta k\left(\frac{p^{q} \log p+(1-p)^{q} \log (1-p)}{p^{q}+(1-p)^{q}}\right)+k O\left(\delta^{2}\right) \\
& -\delta \sum_{i=1}^{k}\left(\varepsilon \mp \alpha_{s}\right)\left|\log r_{i}\right|
\end{aligned}
$$

Upon recalling the definition of $\alpha_{s}$ and noting that $\frac{1}{k} \sum_{i=1}^{k}\left|\log r_{i}\right| \geq \log 2$, the first claim is proved.

If $t=\limsup \frac{1}{k} \sum_{i=1}^{k}\left|\log r_{i}\right|$, then given any $\varepsilon_{1}>0$, for all but finitely many $k$ we have $\frac{1}{k} \sum_{i=1}^{k}\left|\log r_{i}\right| \leq t+\varepsilon_{1}$, and for infinitely many $k, \frac{1}{k} \sum_{i=1}^{k}\left|\log r_{i}\right|$ $\geq t-\varepsilon_{1}$. Because $t \geq 0$ we have $\alpha_{t} \geq 0$, and thus for suitably small $\varepsilon_{1}$ and $\delta$,

$$
\Phi_{ \pm}\left(\alpha_{t}\right) \leq k\left(\mp \alpha_{t} t \delta+O\left(\delta^{2}\right)-\varepsilon \delta \log 2 \pm \alpha_{t} \delta\left(t \pm \varepsilon_{1}\right)\right) \leq-k \delta c \varepsilon
$$

for all but finitely many $k$ in the case of $\Phi_{+}$and for infinitely many $k$ for $\Phi_{-}$.

The argument is similar if $t=\liminf \frac{1}{k} \sum_{i=1}^{k}\left|\log r_{i}\right|$.

Proof of Theorem 16. Let $\alpha \in \mathbb{R}$, fix $\varepsilon>0$ and let $\delta>0$ be small. Temporarily fix $k$ and let $I_{w}(x)$ denote the interval of step $k$ containing $x$. 
Then

$$
\begin{aligned}
v\left\{x: \mu\left(I_{w}(x)\right)\right. & \left.\geq\left|I_{w}(x)\right|^{\alpha-\varepsilon}\right\} \leq \int \mu\left(I_{w}(x)\right)^{\delta}\left|I_{w}(x)\right|^{-(\alpha-\varepsilon) \delta} d v(x) \\
& =\sum_{|w|=k} \mu\left(I_{w}\right)^{\delta}\left|I_{w}\right|^{-(\alpha-\varepsilon) \delta} v\left(I_{w}\right) \\
& =\prod_{i=1}^{k}\left(p^{q+\delta}+(1-p)^{q+\delta}\right) r_{i}^{\beta_{i}+(\varepsilon-\alpha) \delta}=\exp \Phi_{+}(\alpha) .
\end{aligned}
$$

Similarly, $v\left\{x: \mu\left(I_{w}(x)\right) \leq\left|I_{w}(x)\right|^{\alpha+\varepsilon}\right\} \leq \exp \Phi_{-}(\alpha)$.

First, let $t=\limsup \frac{1}{k} \sum_{i=1}^{k}\left|\log r_{i}\right|$. Then $\alpha_{t}=\alpha^{*}$ and hence from the lemma $v\left\{x: \mu\left(I_{w}(x)\right) \geq\left|I_{w}(x)\right|^{\alpha^{*}-\varepsilon}\right\} \leq \exp (-k \delta c \varepsilon)$ for all but finitely many $k$, say for $k \geq k_{1}$. This means that

$$
\sum_{k \geq k_{1}} v\left\{x: \mu\left(I_{w}(x)\right) \geq\left|I_{w}(x)\right|^{\alpha^{*}-\varepsilon}\right\} \leq \sum_{k \geq k_{1}} \exp (-k \delta c \varepsilon)<\infty,
$$

and therefore the probability that $\mu\left(I_{w}(x)\right) \geq\left|I_{w}(x)\right|^{\alpha^{*}-\varepsilon}$ infinitely often is zero. In other words, for $v$ a.e. $x, \mu\left(I_{w}(x)\right) \leq\left|I_{w}(x)\right|^{\alpha^{*}-\varepsilon}$ eventually. Since $\varepsilon>0$ was arbitrary $\liminf \frac{\log \mu\left(I_{w}(x)\right)}{\log \left|I_{w}(x)\right|} \geq \alpha^{*}$ for $v$ a.e. $x$.

Similarly, for infinitely many $k$, say $k \in\left\{k_{n}\right\}$, the lemma shows that

$$
v\left\{x: \mu\left(I_{w}(x)\right) \leq\left|I_{w}(x)\right|^{\alpha^{*}+\varepsilon}\right\} \leq \exp (-k \delta c \varepsilon) .
$$

Thus

$$
\sum_{k_{n}} v\left\{x: \mu\left(I_{w}(x)\right) \leq\left|I_{w}(x)\right|^{\alpha^{*}+\varepsilon}\right\} \leq \sum_{k_{n}} \exp \left(-k_{n} \delta c \varepsilon\right)<\infty
$$

and so for infinitely many $k$ and $v$ a.e. $x$ we have $\mu\left(I_{w}(x)\right) \geq\left|I_{w}(x)\right|^{\alpha^{*}+\varepsilon}$. Consequently,

$$
\underline{\operatorname{dim}}_{l o c} \mu(x)=\liminf \frac{\log \mu\left(I_{w}(x)\right)}{\log \left|I_{w}(x)\right|}=\alpha^{*} \text { for } v \text { a.e. } x .
$$

As $X_{\alpha^{*}}=\left\{x: \underline{\operatorname{dim}}_{l o c} \mu(x)=\alpha^{*}\right\}$ it follows that $v\left(X_{\alpha^{*}}\right)=1$.

Using $t=\liminf \frac{1}{k} \sum_{i=1}^{k}\left|\log r_{i}\right|$ and $\alpha_{t}=\alpha_{*}$, we similarly obtain that

$$
\limsup \frac{\log \mu\left(I_{w}(x)\right)}{\log \left|I_{w}(x)\right|}=\alpha_{*} \text { for } v \text { a.e. } x \text {. }
$$


Thus we also have $v\left(X^{\alpha_{*}}\right)=1$ and hence $v\left(X_{\alpha^{*}} \bigcap X^{\alpha_{*}}\right)=1$.

Recall that

$$
\underline{\operatorname{dim}}_{l o c} v(x) \geq \liminf q \frac{\log \mu\left(I_{w}(x)\right)}{\log \left|I_{w}(x)\right|}+C_{q}
$$

and

$$
\overline{\operatorname{dim}}_{l o c} v(x) \leq \lim \sup q \frac{\log \mu\left(I_{w}(x)\right)}{\log \left|I_{w}(x)\right|}+C^{q} .
$$

Thus if $q>0$, then $\operatorname{\operatorname {dim}}_{l o c} v(x) \geq q \alpha^{+}+C_{q}$ and $\overline{\operatorname{dim}}_{l o c} v(x) \leq q \alpha^{-}+C^{q}$ for $v$ a.e. $x \in X_{\alpha^{*}} \cap X^{\alpha_{*}}$. If $q<0$ the roles of $\alpha^{*}$ and $\alpha_{*}$ are interchanged.

Corollary 18. If $q \geq 0$, then

$$
\begin{aligned}
& \operatorname{dim}_{H}\left(X_{\alpha^{*}} \bigcap X^{\alpha_{*}}\right) \geq q \alpha^{*}+C_{q}, \\
& \operatorname{dim}_{P}\left(X_{\alpha^{*}} \bigcap X^{\alpha_{*}}\right) \leq q \alpha_{*}+C^{q} .
\end{aligned}
$$

If $q<0$, then

$$
\begin{aligned}
& \operatorname{dim}_{H}\left(X_{\alpha^{*}} \bigcap X^{\alpha_{*}}\right) \geq q \alpha_{*}+C_{q} \\
& \operatorname{dim}_{P}\left(X_{\alpha^{*}} \bigcap X^{\alpha_{*}}\right) \leq q \alpha^{*}+C^{q} .
\end{aligned}
$$

Proof. This is immediate from Theorem 16 upon applying the mass distribution principle (see [4], 2.3).

If, in addition, $\lim _{k}\left|\frac{1}{k} \log r_{1} \cdots r_{k}\right|=\tau$, then $\tau=\log 2 / \operatorname{dim}_{H} C$,

$$
C^{q}=C_{q}=\log \left(p^{q}+(1-p)^{q}\right) / \tau
$$

and

$$
\alpha^{*}=\alpha_{*}=\frac{-\left(p^{q} \log p+(1-p)^{q} \log (1-p)\right)}{\left(p^{q}+(1-p)^{q}\right) \tau} \equiv \alpha(q) .
$$

Consequently, for all $x \in X(\alpha(q))=\left\{\operatorname{dim}_{l o c} \mu(x)=\alpha(q)\right\}$,

$$
\operatorname{dim}_{l o c} v(x)=q \alpha+\frac{\log \left(p^{q}+(1-p)^{q}\right)}{\tau} .
$$

This gives the formulas

Corollary 19. If $\lim _{k}\left|\frac{1}{k} \log r_{1} \cdots r_{k}\right|$ exists, then

$$
\operatorname{dim}_{H} X(\alpha(q))=\operatorname{dim}_{P} X(\alpha(q))=q \alpha+\frac{\log \left(p^{q}+(1-p)^{q}\right) \operatorname{dim}_{H} C}{\log 2} .
$$


Finally, consider the Legendre transform of the function $C_{q}$ (under the assumption that $\lim _{k}\left|\frac{1}{k} \log r_{1} \cdots r_{k}\right|$ exists):

$$
f(\alpha)=\inf _{q}\left(q \alpha+C_{q}\right)=\inf _{q}\left(q \alpha+\frac{\log \left(p^{q}+(1-p)^{q}\right)}{\tau}\right) .
$$

A simple calculus argument shows that the infimum is attained when $q$ is such that $\alpha=\alpha(q)$. Thus $\operatorname{dim}_{H} X(\alpha(q))=f(\alpha(q))$. Furthermore, observe that every value in the interval $[-\log p / \tau,-\log (1-p) / \tau]$ is of the form $\alpha(q)$ for some $q \in \mathbb{R}$. Thus the multifractal spectrum of $\mu$ (i.e., the dimension of $X(\alpha)$ ) is given by the Legendre transform of the multifractal $q$-box dimensions.

Corollary 20. Let $C$ be a Cantor set and suppose $\mu$ is the p-Cantor measure. Assume $\tau=\lim _{k}\left|\frac{1}{k} \log r_{1} \cdots r_{k}\right|$ exists. Then for every

$$
\alpha \in\left[\frac{-\log p}{\tau}, \frac{-\log (1-p)}{\tau}\right],
$$

we have

$$
\operatorname{dim}_{H} X(\alpha)=\operatorname{dim}_{P} X(\alpha)=f(\alpha)
$$

where $f$ is the Legendre transform of the function which gives the multifractal $q$-box dimensions: $q \longmapsto \log \left(p^{q}+(1-p)^{q}\right) / \tau$.

Remark 1. This improves upon results in [8] since we have seen in Prop. 4 that such Cantor measures need not be quasi self-similar.

\section{References}

[1] Cabrelli, C., Hare, K. and Molter, U., Sums of Cantor sets, Ergodic Th. and Dynamical Systems 17(1997), 1299-1313.

[2] Cawley, R. and Mauldin, R.D., Multifractal decomposition of Moran fractals, Advances in Math. 92(1992), 196-236.

[3] Edgar, G.D. and Mauldin, R.D., Multifractal decompositions of digraph recursive fractals, Proc. London Math. Soc. 65(1992), 604-628.

[4] Falconer, K., Techniques in fractal geometry, Wiley and Sons, Chichester (1997).

[5] King, J., The singularity spectrum for general Sierpinski carpets, Advances in Math. 116(1995), 1-11. 
[6] Olsen, L., A multifractal formalism, Advances in Math. 116(1995), 82-196.

[7] Olsen, L., Multifractal dimensions of product measures, Math. Proc. Camb. Phil. Soc. 120(1996), 709-734.

[8] O'Neil, T., The multifractal spectrum of quasi self-similar measures, J. Math. Anal. and Appl. 211(1997), 233-257.

[9] Rand, D., The singularity spectrum $f(\alpha)$ for cookie cutters, Ergodic Th. Dynamical Sys. 9(1989), 527-541. 
Kathryn E. Hare and Saroosh Yazdani 